
\title{
25 Research Square \\ Label-Free Detection of Human Coronaviruses in Infected Cells Using Enhanced Darkfield Hyperspectral Microscopy (EDHM)
}

Devadatta Gosavi

Auburn University

Byron Cheatham

Cytoviva, Inc

Joanna Sztuba-Solinska ( jzs0165@auburn.edu )

Auburn University

\section{Research Article}

Keywords: Human coronaviruses (HCoV), enhanced darkfield hyperspectral microscopy (EDHM), uninfected cells

Posted Date: October 29th, 2021

DOI: https://doi.org/10.21203/rs.3.rs-1000311/v1

License: (c) (i) This work is licensed under a Creative Commons Attribution 4.0 International License. Read Full License

Version of Record: A version of this preprint was published at Journal of Imaging on January 25th, 2022. See the published version at https://doi.org/10.3390/jimaging8020024. 


\section{Abstract}

Human coronaviruses (HCoV) are causative agents of mild to severe intestinal and respiratory infections in humans. In the last 15 years, we have witnessed the emergence of three zoonotic, highly pathogenic HCoVs. Thus, early, and accurate detection of these viral pathogens is not only essential for preventing transmission but also for the timely treatment and monitoring of drug resistance. Herein, we applied enhanced darkfield hyperspectral microscopy (EDHM), a novel non-invasive, label-free diagnostic tool for rapid and accurate identification of two strains of HCoVs, i.e., OC43 and 229E. The EDHM technology allows collecting the optical image with both spectral and spatial details in a single measurement without direct contact between the specimen and the sensor. Thus, it can provide the direct mapping of spectral signatures specific for a given viral strain in a complex biological milieu. Our study demonstrated distinct spectral patterns for HCoV-OC43 and HCoV-229E virions in the solution, which can serve as distinguishable parameters for their differentiation. Furthermore, spectral signatures for both HCoV strains in the infected cells displayed a considerable peak wavelength shift compared to the uninfected cell samples indicating that the EDHM is applicable to detect and differentiate between HCoV infected and uninfected cells.

\section{Introduction}

Viruses belonging to different families pose a continuous threat to public health and worldwide stability. The unprecedented outbreaks of severe acute respiratory syndrome-associated coronavirus (SARS-CoV) ${ }^{1}$, Middle East respiratory syndrome coronavirus (MERS-CoV) ${ }^{2}$, and the ongoing pandemic of severe acute respiratory syndrome-associated coronavirus 2 (SARS-CoV-2) ${ }^{3}$ poised human coronaviruses to take prominence on the world stage, highlighting the necessity for readily available, accurate, and fast diagnostic testing methods.

Coronaviruses are positive-sense single-stranded RNA viruses that belong to the family Coronaviridae, which includes four genera, several sub-genera, and species. Four human coronaviruses (HCoVs) are globally endemic, including two Alphacoronavirus (HCoV-229E and HCoV-NL63), and two Betacoronavirus (HCoV-OC43 and HCoV-HKU1). These HCoVs account for $10-30 \%$ of upper respiratory tract infections in adults ${ }^{4}$. The emergence of HCoV-229E was estimated to occur about 200 years ago, and it involved the transfer from bats to alpacas and then to humans. About 120 years ago, HCoV-OC43, which was considered to have a common ancestor with bovine coronavirus, was transmitted from cattle to humans ${ }^{5}$ Subsequently, both HCoV-229E and HCoV-OC43 were isolated from the nasal cavities of people with the common cold in the $1960 s^{6}$. In the 1970s, studies that used serology and viral culture linked HCoVs-229E and -OC43 with 8\% of cases of lower respiratory tract infection in hospitalized infants ${ }^{7}$. HCoV-NL63 and HCoV-HKU1 were discovered in the early 2000s from persons with bronchiolitis and pneumonia ${ }^{8,9}$. In 2002 a Betacoronavirus originating in bats, then spread from civets to humans causing severe respiratory disease and taking the name SARS-CoV ${ }^{10}$. In 2012 a Betacoronavirus spread from camels to humans in Saudi Arabia, causing a similar clinical syndrome as SARS, taking the name 
MERS-CoV ${ }^{11}$. The latest SARS-CoV-2 is closely related to Betacoronaviruses detected in bats $(88 \%$ sequence identity) but is genetically distinct from SARS-CoV ( 79\% sequence identity $)^{12}$. It is understood that a broad host range ${ }^{13}$, frequent cross-species transmission, stability of the virus in the environment, and changes in the tissue tropism ${ }^{14}$, support the emergence of new highly infectious human coronaviruses.

There is a general lack of adequate clinical characteristics and epidemiological data available on human coronaviruses. This knowledge gap primarily persists due to the difficulties in developing cellular and animal model systems to uncover the mechanisms of viral pathogenicity and design adequate therapeutic strategies ${ }^{15}$. Also, a critical deficiency lies in the available diagnostic tests that should allow for early, rapid, and sensitive detection of coronaviruses. Quantitative reverse transcriptase-polymerase chain reaction (RT-qPCR) is currently the most frequently used technique to detect viral nucleic acids present in bodily fluids. Testing facilitates the prevention of spread between persons and communities that include asymptomatic infected people, whose viral shedding can unintentionally spread the infection to the elderly and those that are immunocompromised. However, the total turnaround time for RT-qPCR can exceed two days, can be burden with false-negative results caused by a low viral load or a presence of genetic variants of the virus ${ }^{16}$, and false-positive results arising from cross-contamination ${ }^{17}$. Serological testing complements virus detection, indicating past infection, which could be harnessed for therapeutic gain ${ }^{18}$. Here, antibodies are detected by enzyme-linked immunosorbent assay using a qualitative detection of IgG or IgM antibodies ${ }^{19}$. Such tests determine an immune response against the viral antigen and may be helpful to assess protection against subsequent viral exposure and for contact tracing purposes. However, serological tests usually detect the antibodies and not the virus itself, and the effectiveness of these assays during the early stages of infection when adaptive immunity is building is therefore limited ${ }^{20}$. Fluorescence and confocal microscopies are widely employed for single-virus tracking, as they allow visualization of particular specimen in the complex biological environment in a minimally invasive manner. However, the analyses are time-consuming, require fluorescent labels for single-virus tracking, and a good portion of the sample is always illuminated leading to photobleaching of the sample even out of the focal plane ${ }^{21}$. In response to the limitations of the above-mentioned diagnostic methods, new platforms are actively being pursued.

In this manuscript, we outline the advantages of using the enhanced darkfield hyperspectral microscopy (EDHM) as a novel tool that allows a label-free detection of two HCoV strains, i.e., OC43 and 229E in infected mammalian cells. The technology (developed by CytoViva, Inc. Auburn, AL, USA) offers the high contrast visualization of low contrast objects, which are typically not visible by conventional brightfield microscopy. These factors are of great interest for the microscopic examination of biological samples, as the technology does not require the application of contrasting agents. When combined with hyperspectral imaging, the optical image with both spectral and spatial details can be acquired in a single measurement without direct contact between the specimen and the sensor ${ }^{22}$. The integrated system can provide the direct mapping of spectral signatures specific for a given viral strain in a complex biological milieu based on its unique optical spectrum. Accordingly, this method is sensitive to subtle spectral 
changes, ensuring thorough discrimination of biological entities or changes in tracking agents over time ${ }^{23,24}$. As such, EDHM represents a novel alternative to the available diagnostic tools for the detection of various infectious agents.

\section{Materials And Methods}

Cell lines and culture conditions. Human colon adenocarcinoma (HCT-8 cells, ATCC CCL-244) were seeded in $75 \mathrm{~cm}^{2}$ cell culture flasks at $4.7 \times 10^{6} \mathrm{cells} / \mathrm{ml}$ and grown in RPMI-1640 medium (ATCC 30-2001) supplemented with $10 \%$ horse serum (HS) (Fisher scientific SH3007403) and $1 \mathrm{X}$ Penicillin/Streptomycin/Glutamine (Gibco 10378016) at $37^{\circ} \mathrm{C}$ in $5 \% \mathrm{CO}_{2}$. Medical research council cells strain 5 (MRC-5 cells, ATCC CCL-171) were seeded in $75 \mathrm{~cm}^{2}$ cell culture flasks at $1 \times 10^{6}$ cells $/ \mathrm{ml}$ and grown in Eagle's Minimum Essential Medium (EMEM, ATCC 30-2003) supplemented with $10 \%$ fetal bovine serum (FBS) (ATCC 30-2020), 1X Penicillin/Streptomycin/Glutamine (Gibco, 10378016) at $37^{\circ} \mathrm{C}$ in $5 \% \mathrm{CO}_{2}$. The monolayers of both HCT-8 or MRC- 5 cells were washed with $1 \mathrm{X}$ Phosphate-Buffered Saline (PBS, Corning 21-040-CM) and treated with Trypsin-EDTA solution (VWR L0154-0100) for $5 \mathrm{~min}$ at $37^{\circ} \mathrm{C}$ in $5 \% \mathrm{CO}_{2}$ to set up subculture every 2-3 days.

Coronavirus Propagation. HCoV-OC43 and HCoV-229E viral stocks were originally obtained from ATCC (VR-1558 and VR-740 respectively). The 80 - 90\% confluent HCT-8 cells ( $6 \times 10^{6}$ cells $\left./ \mathrm{ml}\right)$ were transfected with HCoV-OC43 at a viral titer of $1.12 \times 10^{5} \mathrm{PFU} / \mathrm{ml}$ (multiplicity of infection, MOI 0.05). The $80-90 \%$ confluent MRC- 5 cells $\left(\sim 5.4 \times 10^{6}\right.$ cells $\left./ \mathrm{ml}\right)$ were transfected with HCoV-229E at a viral titer of $1.12 \times 10^{6} \mathrm{PFU} / \mathrm{ml}(\mathrm{MOI} 0.5)$. The viral adsorption was performed in a minimum volume of serum-free media to facilitate the efficient diffusion of viral particles into the target cells. The HCT-8 cells were incubated for $1 \mathrm{~h}$ at $33^{\circ} \mathrm{C}$, while MRC- 5 cells were incubated for $1 \mathrm{~h}$ at $35^{\circ} \mathrm{C}$ with continuous rocking. Viral suspensions were aspirated and RPMI- 1640 medium containing $2 \% \mathrm{HS}$ was added to HCoV-OC43 infected cell culture and incubated for 11 days at $33^{\circ} \mathrm{C}$ in $5 \% \mathrm{CO}_{2}$, while $\mathrm{HCoV}-229 \mathrm{E}$ infected cells were incubated with EMEM medium containing $2 \% \mathrm{FBS}$ media for 5 days at $35^{\circ} \mathrm{C}$ in $5 \% \mathrm{CO}_{2}$. Cell morphology was monitored daily to observe for any cytopathic effects by bright-field microscopy.

Preparation of viral stocks. Following 11- or 5- days post-infection for HCT-8 and MRC-5 cell lines, respectively, the cells were harvested, and virions were released by four freeze-thaw cycles in respective maintenance media (serum-free). The suspensions were clarified by centrifugation at $1500 \mathrm{xg}$ for $15 \mathrm{~min}$ at $0^{\circ} \mathrm{C}$, and the supernatant was mixed with $8.3 \%$ polyethylene glycol (PEG, VWR 97061-102) with rapid mixing and on ice. The solution was incubated at $+4^{\circ} \mathrm{C}$ overnight, followed by centrifugation at $1800 \mathrm{xg}$ for $30 \mathrm{~min}$ at $0^{\circ} \mathrm{C}$. The virus pellets were resuspended in $500 \mu \mathrm{NET}$ buffer $(0.15 \mathrm{M} \mathrm{NaCl}, 0.0005 \mathrm{M}$ EDTA, $0.02 \mathrm{M}$ Tris, $\mathrm{pH} 7.2$ ) and stored at $-80^{\circ} \mathrm{C}$.

Plaque Assays. $1 \times 10^{6}$ cells (HCT-8 and MRC-5) were seeded in a 6-well cell culture plate to achieve 80 $90 \%$ confluency after $24 \mathrm{~h}$. The monolayers were washed twice with 1X PBS. Viral samples were diluted 10 -fold in respective maintenance media (serum-free) and the appropriate dilution was added to the 
corresponding wells. A single well containing uninfected control cells was maintained for monitoring the cellular viability and plaque detection. The viral adsorption was carried out for $1 \mathrm{~h}$ at $33^{\circ} \mathrm{C}$ for $\mathrm{HCoV}-\mathrm{OC} 43$ and $35^{\circ} \mathrm{C}$ for $\mathrm{HCoV}-229 \mathrm{E}$ in $5 \% \mathrm{CO}_{2}$ with intermittent rocking of the plates for $20 \mathrm{~min}$ to ensure even virions adsorption and to prevent the monolayer from drying. The overlay medium was prepared by combining $0.6 \%$ agarose (VWR Life Science, 97062-244) with respective maintenance media. $2 \mathrm{ml}$ of overlay medium was added to each well, and the plates were incubated for 11 days at $33^{\circ} \mathrm{C}$ in $5 \% \mathrm{CO}_{2}$ for $\mathrm{HCoV}-\mathrm{OC} 43$ infected cells or 5 days at $35^{\circ} \mathrm{C}$ in $5 \% \mathrm{CO}_{2}$ for HCoV-229E infected cells (Figure 5).

Fixation and staining. After incubation, $200 \mu \mathrm{l}$ of $4 \%$ paraformaldehyde in a $0.1 \mathrm{M}$ cacodylate buffer solution (Fischer Scientific 50-190-1157) was applied directly to each well followed by incubation for $1 \mathrm{~h}$ at room temperature. The fixing solution was aspirated, and agarose plugs were removed. The cells were stained using $1 \%$ crystal violet solution (Millipore Sigma V5265) for 10 min with gentle rocking. The crystal violet solution was removed, and plates were dried to estimate the plaques count. The virus titer was determined according to the following formula: Virus titer $(\mathrm{pfu} / \mathrm{ml})=$ average number of plaques $/$ (dilution factor * volume of diluted virus added to the well).

Preparation of cell and viral samples for EDHM. $0.17 \mathrm{~mm}$ glass coverslips (Fischer Scientific, 10474379) were coated with $800 \mu \mathrm{l}$ gelatin (Millipore sigma, G1393) and incubated for $2 \mathrm{~h}$ at room temperature. The gelatin was aspirated, and coverslips were washed with 1X PBS and dried for $3 \mathrm{~h}$ at room temperature. The 24-well cell culture plate was seeded at $\sim 5 \times 10^{5}$ cells $/ \mathrm{ml}$ uninfected or infected cells (MRC- 5 or HCT8 ) in the respective maintenance medium. $24 \mathrm{~h}$ later, the cells were washed with $1 \mathrm{XPBS}$, fixed with $4 \%$ $800 \mu \mathrm{l}$ paraformaldehyde in $0.1 \mathrm{M}$ cacodylate buffer solution, and incubated for $15 \mathrm{~min}$ at room temperature. The cells were washed with 1X PBS twice prior mounting the coverslips on the slides. For the preparation of the virus samples, $5 \mu$ of the viral stock solution $\left(1.12 \times 10^{5} \mathrm{pfu} / \mathrm{ml}\right.$ of HCoV-OC43 or $1.12 \times 10^{6} \mathrm{pfu} / \mathrm{ml}$ of HCoV-229E) were incubated with $5 \mu 4 \%$ formaldehyde in $0.1 \mathrm{M}$ cacodylate buffer solution for $15 \mathrm{~min}$ at room temperature and mixed with $6 \mu \mathrm{l}$ mounting media ( $50 \%$ glycerol, $0.1 \mathrm{M}$ Tris, pH 8.5). The viral samples were placed on a glass slide (Fischer Scientific, 12-549-3) with a coverslip and directed to dark field microscopy analysis.

EDHM Imaging of HCoV viral samples. The stock or PEG precipitated viral samples (HCoV-OC43 or HCoV229E) were visualized using CytoViva EDHM imaging system mounted on a standard research-grade optical microscope with an oil immersion darkfield illuminator which was adjusted in the $X, Y$, and $Z$ focal range using a 10X objective and a condenser. The optical images of viral samples were observed using a $60 \mathrm{x}$ oil adjustable iris objective with the numerical aperture (NA) set to 1.2NA and the tungsten halogen light source adjusted to 150 watts power. The hyperspectral images were captured with $2 x$ binning of the camera with an exposure of 0.7 seconds for the 229E sample and 0.4 seconds for the OC43 sample producing a total image pixel resolution of $700 \times 300$ for each sample. Based on the hyperspectral data set, the image analysis at each pixel can be obtained using the CytoViva software program (ENVI 4.8). In this process, the hyperspectral image analysis particle filter feature was utilized to identify the viral particles in each sample, and the pixel level spectrum obtained from each of these identified particles 
was then grouped together as a region of interest $(\mathrm{ROI})$. The mean spectral analysis algorithm was then employed to create a mean spectral response from these ROls and the mean spectrum was normalized to a peak intensity of one for comparative analysis using a preset spectral math equation that is built into the spectral analysis software.

EDHM imaging of mammalian cells infected with HCoV. Similar to the imaging of viral samples, EDHM images for mammalian cells infected with HCoV-OC43 or HCoV-229E were visualized using a 60x oil iris objective with the numerical aperture set 1.2 NA and quartz halogen light source adjusted to 150 watts power. The hyperspectral images were captured with an exposure of 0.25 seconds and $2 x$ binning of the camera. After scanning the entire area of each cell infected with the virus, different areas with suspected viral particles were captured with EDHM and hyperspectral data cubes (hyperspectral images which can be analyzed by spectral angle mapper (SAM)) were captured of the same areas for spectral mapping. Both uninfected mammalian cells (negative control) and cells infected with HCoV-OC43 or HCoV-229E were imaged following the same steps.

For hyperspectral mapping analysis, the HCT8 cell samples infected with OC43 virus were scanned for large areas of individual pixel level spectrum and were grouped together in regions of interest (ROIs). Each of these ROls were then converted to spectral libraries representing thousands of individual pixel spectral data obtained from each pixel of ROI. Individual SLs obtained from the cells infected with coronavirus were then filtered against the negative control data cubes using the Filter Spectral Library algorithm for removing any duplicative spectra and for creating a reference spectral library (RSL). The RSL was then used to map against all the data cubes obtained from the cells infected with OC43 for detecting the areas with the same spectral profile as the RSL using the SAM function in the ENVI 4.8 software. The mapped areas

were merged in one color and overlaid on the original data cube to observe where the viral particles of interest were located within each sample. A similar process outlined above was performed on the MRC5 cells exposed to the $229 \mathrm{E}$ virus.

\section{Results}

The overview of enhanced darkfield hyperspectral microscopy (EDHM). EDHM is specifically designed for imaging and analysis of a wide range of materials and biological samples in situ. The use of tungstenhalogen light (Dolan Jenner, Boxborough, MA) as a source of illumination generates a spectral output from 400 to 2,200 $\mathrm{nm}$. The indirect illumination upon interaction with the sample collects the reflected or elastically scattered light ${ }^{25}$, which permits distinguishable visualization of objects with similar refractive indexes as the background. The enhanced darkfield illuminator system consists of an annular cardioid condenser, which produces highly collimated light at oblique angles. This generates images of samples with enhanced contrast and 10 times higher signal-to-noise ratio compared to conventional darkfield optics $^{23}$. The image from the scattered light is projected onto a visible and near-infrared (VNIR) diffraction grating spectrograph (Specim, Oulu, Finland) and captured using a regular charge-coupled 
device (CCD) video camera (PCO, Kelheim, Germany). The hyperspectral image is subsequently analyzed using ENVI 4.8 hyperspectral image analysis software, which generates unique spectral signatures for individual pixels of the analyzed sample and saves it as a Reference Spectral Library (RSL). The spectral response characteristic of the biological sample is subsequently mapped using Spectral Angle Mapper (SAM), which is a powerful algorithm utilized to determine the spectral resemblance between two spectral profiles and to match the pixels to reference spectrum ${ }^{26}$.

Propagation and quantification of human coronaviruses, HCoV-OC43 and HCoV-229E. To demonstrate the applicability of EDHM for the differential detection of human coronaviruses, we acquired two commercially available strains, i.e., HCoV-OC43 and HCoV-229E, that were used to infected HCT-8 and MRC- 5 cell lines at MOIs of 0.05 and 0.5 , respectively ${ }^{27}$. The cytopathic effect (CPE) for HCoV-OC43 was detectable 11 days post-infection, and involved rounding-up and detachment of cells, while the CPE for HCoV-229E infected cells was observable following day 5 and involved cell spindling and detachment. Subsequently, the virions were isolated using freeze-thaw cycles performed in respective maintenance media, followed by overnight precipitation in the presence of high molecular weight polyethylene glycol solution (PEG). High molecular weight PEG has been widely used for the isolation of many RNA viruses, including influenza virus ${ }^{28}$, respiratory syncytial virus ${ }^{29}$, and DNA viruses, e.g., bacteriophages ${ }^{30}$ owing to its simplicity and capability to precipitate viruses at neutral $\mathrm{pH}$ and high ionic concentrations ${ }^{31}$. Following precipitation, the concentration of viral particles was assessed using plaque assays (Figure 1). The viral titers were calculated by dividing the average number of plaques by the dilution factor. For HCoV-OC43 the titer was estimated as $7.5 \times 10^{5} \mathrm{PFU} / \mathrm{ml}$, while for $\mathrm{HCoV}-229 \mathrm{E}$, it was estimated as $6 \times 10^{5} \mathrm{PFU} / \mathrm{ml}$.

EDHM analysis of HCoV-OC43 and HCoV-229E virions. The hyperspectral images of three biological replicates of commercially obtained and prepared viral stocks of HCoV-OC43 and HCoV-229E were captured using EDHM with the numerical aperture of the 60X objective set to 1.2NA and hyperspectral camera exposure time set to 0.4 seconds for $\mathrm{HCoV}-\mathrm{OC} 43$ and 0.7 seconds for $\mathrm{HCoV}-229 \mathrm{E}$ samples. The obtained hyperspectral images had the full VNIR spectral data ranging from $400-1,000 \mathrm{~nm}$ for each pixel with $2 \mathrm{~nm}$ spectral resolution across the full VNIR wavelength range. The use of enhanced darkfield light illumination optics improved the scatter properties of the virus particles, which contributed to optimum visualization of individual viral particles in the hyperspectral image.

The analysis of hyperspectral images of viral particles in solution was performed using the mean spectral analysis algorithm, which generates mean spectral responses for individual pixels. The mean spectra were normalized to a peak intensity of 1 , and the spectral responses for the stock and precipitated HCoVOC43, and HCoV-229E samples were compared within the same graph. The analysis displayed almost identical spectral profiles obtained for the commercially acquired and prepared virions of HCoV-OC43 and HCoV-229E (Figure 2). However, the mean spectral comparisons of the HCoV-OC43 and HCoV-229E samples demonstrated the different spectral responses with $\sim 30 \mathrm{~nm}$ shift of the spectral peaks. This is an important distinguishable feature that can be used for differential mapping and detection of both coronavirus strains. 
EDHM analysis of HCT-8 and MRC-5 infected cell lines. We have also performed EDHM analysis of uninfected and infected mammalian cells (HCT-8 and MRC-5) to measure how the cellular milieu would affect the spectral profiles of analyzed coronavirus strains. The images of three independent biological replicates of uninfected and infected HCT-8 and MRC- 5 cells were captured using a hyperspectral camera with a detection range of $400-1000 \mathrm{~nm}$, a $60 x$ microscope objective magnification with a $6.4 \mu \mathrm{m}$ camera pixel size and $2 x$ pixel binning. In the resulting images, each pixel consists of the spectral profile of the sample at the corresponding spatial position and can be used to detect the viral particles with neardiffraction-limited resolution $(1 \mu \mathrm{m})$. The RSL for the infected cells was created by filtering out the spectrum obtained for uninfected cells, which was regarded as a background using the Filter Spectral algorithm. The RSLs were used to map hyperspectral images by employing the SAM function in the ENVI 4.8 software, which allows for the location and identification of viral particles within the infected cell.

The mean spectral profiles of uninfected HCT-8 and MRC- 5 cells, and cells infected with HCoV-OC43 and HCoV-229E, respectively, revealed unique spectral mappings, which correspond to viral particles detected within the cellular milieu. The hyperspectral image analysis of HCT-8 cells infected with HCoV-OC43 resulted in a spectral peak of $\sim 575 \mathrm{~nm}$, which is in contrast to uninfected cells that resulted in a spectral peak of $\sim 525 \mathrm{~nm}$ (Figure 3). Also, we observed a significant difference in the optical spectral response for the mapped areas of HCoV-229E infected MRC- 5 cells with a spectral peak at $\sim 650 \mathrm{~nm}$ compared to uninfected MRC5 cells with a spectral peak at $\sim 500 \mathrm{~nm}$ (Figure 4). This indicates that the EDHM is applicable to detect and differentiate between infected and uninfected cells.

\section{Discussion}

Recognition of the importance of community diseases caused by human coronaviruses has increased in recent years; however, detailed information on pathogenesis, immunity, and viral characteristics remains limited. As such, significant efforts have been made to develop more sensitive diagnostic tools and molecular detection methods ${ }^{32}$. In this manuscript, we described the application of a label-free method for direct visualization and relative quantitative analysis of two human coronaviruses, i.e., HCoV-OC43 and HCoV-229E in a cellular environment by combining the enhanced darkfield microscopy and hyperspectral mapping. First, using hyperspectral image analysis we mapped the commercially acquired and prepared viral samples and compared their spectral profiles to verify whether the PEG precipitated virions display the same spectral characteristic as the commercially acquired samples. Here, we found that their spectral signatures were identical. Next, we compared the mean spectral profiles of HCoV-OC43 with HCoV-229E virions in the solution and noted a distinct spectral signal for each viral strain. Thus, these spectral profiles can be used as a distinguishable parameter for the differentiation of these two human coronavirus strains. Further, the spectral profiles of uninfected and infected cells were compared to understand to what extent the spectral signature of virions would be detectable in the cellular environment. Here, we noted that the spectral signatures of the infected cells exhibited a considerable peak shift compared to the uninfected cells. As the spectral profiles for HCoV-OC43 and HCoV-229E in the infected cells are distinct from each other, these unique spectral responses from the virions can be further 
mapped in the infected cells and utilized for the generation of the reference libraries for future analysis. Thus, the application of EDHM analysis allows differentiation between two HCoV strains when present in the solution or cellular milieu.

EDHM offers a significant advantage over conventional imaging techniques, as it involves minimum and non-destructive sample preparation, fast image acquisition, and rapid analysis Additionally, it has the capability to determine the spatial distribution and characterization of samples in complex biological environments ${ }^{33}$. Moreover, EDHM can be operated by a relatively inexperienced individual with minimal training compared to other microscopic techniques, such as transmission electron microscopy (TEM) or scanning electron microscopy (SEM). EDHM system is also significantly less expensive than alternative options with estimated costs for the system averaging at approximately $\$ 155,000$. In comparison, TEM system costs on average $\$ 4.0$ million, while SEM system expenses are near $\$ 1.0$ million $^{24}$.

EDHM has been applied to deliver real-time images of biomarker information and to examine cell pathophysiology depending on the spectral resonance characteristics in relevant tissues. For example, EDHM has been approved for in vitro screening of chemical entities for amyloidogenesis modulatory activity and for detection of $A \beta$ aggregate in Alzheimer's mouse brain and retina by analyzing unique signatures for $A \beta$ plaques ${ }^{34}$. EDHM is also a convenient, noninvasive tool for assessing signs of hemorrhagic shock (HEM), for example, the quantification of changes in the surface tissue saturation of oxygen $\left(\mathrm{S}_{\mathrm{HSI}} \mathrm{O}_{2}\right)$, wherein the brightness of the oxygen saturation images generated through EDHM is proportional to the intensity of $\mathrm{S}_{\mathrm{HSI}} \mathrm{O}_{2}{ }^{35}$. EDHM in combination with an artificial neural network (ANN) has been used for the diagnosis of urolithiasis recidivism ${ }^{36}$, which affects $10-20 \%$ of the population in developed countries ${ }^{37}$. In this case, EDHM provides rapid characterization and classification of renal calculi within the urinary tract compared to conventional techniques like stereoscopic microscopy ${ }^{38}$ and infrared analysis ${ }^{39}$.

Previously an electronic biosensor based on single-walled carbon nanotubes (SWCNTs) network was used for recognition of the dengue virus in infected cells ${ }^{40,41}$. However, numerous studies have indicated that SWCNTs have the potential to cause pulmonary injury $42-45$, and increase the susceptibility of small airway epithelial cells (SAEC) to influenza A virus (IAV) ${ }^{46}$. EDHM has been used to evaluate the unique spectral profiles and cellular localization of SWCNTs and viral particles in fixed SAEC. This analysis suggested that the co-exposure of the viral particles and SWCNTs to SAEC increased the intracellular localization of $\mathrm{IAV}^{46}$.

Recently EDHM has been applied to track gold nanoparticles capped with antisense oligonucleotides (Au$\mathrm{ASO}_{\text {mix }}$ ) against SARS CoV-2. In the presence of SARS-CoV2 infection, a notable number of agglomerated gold nanoparticles were detected indicating the specific binding of nanoparticles to SARS CoV2 RNA. Further, a significant hyperspectral shift and broadening of hyperspectral signatures were also observed for the $\mathrm{Au}-\mathrm{ASO}_{\text {mix }}$ nanoparticles in the presence of viral RNA ${ }^{47}$. 
Besides, a modified version of the EDHM system including the outlier removal auxiliary classifier generative adversarial nets (OR-AC-GAN), has been applied to detect early symptoms of the disease caused by Tomato Spotted Wilt Virus (TSWV). OR-AC-GAN is a popular neural network architecture in the deep learning domain ${ }^{48-50}$, that in combination with EDHM, has been utilized for image segmentation, feature extraction, and spectrum classification. This modified EDHM system can distinguish the pixels of healthy plants and plants infected with TSWV at early stages before the symptoms are visible on the plants, thus facilitating the management and spread of disease ${ }^{51}$. Thus, there is an upward trend in the utilization of EDHM for medical diagnostics, as well as for image-guided surgeries.

Although the EDHM confers various advantages over conventional microscopy techniques, in terms of cost and time reduction, the amount of data generated to form the hypercubes (hyperspectral objects resulting from the hyperspectral measurements of wavelength information for all the bands in a hyperspectral image ${ }^{41}$ requires substantial processing, large data storage, and accurate analysis to extract appropriate conclusive information ${ }^{26,52}$. Occasionally, along with the reflected/scattered light from the sample, the out-of-focus light also reaches the objective, reducing the spatial resolution of the image. Therefore, appropriate instrumental adjustments are required to provide the users with vertical scanning capabilities ${ }^{53}$.

In summary, the EDHM offers a novel diagnostic tool that can be utilized to detect and differentiate infectious agents, e.g., HCoVs in the solution and within infected cells. This technique can also be modified to obtain the real-time images of biomarkers related to virus-induced pathogenesis, e.g., acquired immunodeficiency syndrome (AIDS) which results in dark skin lesions, Eczema herpeticum, and for analyzing the cell pathophysiology based on the spectral characteristics of relevant tissue. This will aid in the early detection of the symptoms and efficient treatment of diseases. Additionally, combining EDHM with other techniques like Raman spectroscopy will improve the diagnostic applications of this technique.

\section{Declarations}

\section{Acknowledgement}

We would like to thank Cytoviva, Inc. for the funding. J.S.S and D.G. are also supported by start-up funds from the Department of Biological Sciences, College of Science and Mathematics, and Office of the Vice President for Research, Auburn University.

\section{Author contributions}

Conceptualization, J.S.S., B.C. ; methodology, D.G.,B.C. ; formal analysis, J.S.S., D.G., B.C. ; writingoriginal draft preparation, J.S.S., D.G., B.C. ; writing-review and editing, J.S.S, B.C. ; visualization, D.G, B.C. ; supervision, J.S.S.; project administration, J.S.S, B.C. ; funding acquisition, J.S.S., B.C. All authors have read and agreed to the published version of the manuscript. 


\section{Competing Interests}

The authors declare no competing financial interests.

\section{Additional information}

Correspondence and requests for materials should be addressed to J.S.S

\section{References}

1. Cheng, V. C. C., Lau, S. K. P., Woo, P. C. Y. \& Kwok, Y. Y. Severe acute respiratory syndrome coronavirus as an agent of emerging and reemerging infection. Clin. Microbiol. Rev.20, 660-694 (2007).

2. Ramadan, N. \& Shaib, H. Middle east respiratory syndrome coronavirus (MERS-COV): A review. Germs9, 35-42 (2019).

3. Yang, Y. et al. SARS-CoV-2: characteristics and current advances in research. Virol. J.17, 1-5 (2020).

4. Van Elden, L. J. R. et al. Frequent Detection of Human Coronaviruses in Clinical Specimens from Patients with Respiratory Tract Infection by Use of a Novel Real-Time Reverse-Transciptase Polymerase Chain Reaction. J. Infect. Dis.189, 652-657 (2004).

5. Forni, D., Cagliani, R., Clerici, M. \& Sironi, M. Molecular Evolution of Human Coronavirus Genomes. Trends Microbiol.25, 35 (2017).

6. Su, S. et al. Epidemiology, Genetic Recombination, and Pathogenesis of Coronaviruses. Trends Microbiol.24, 490-502 (2016).

7. Mclntosh, K. et al. Coronavirus infection in acute lower respiratory tract disease of infants. J. Infect. Dis.130, 502-507 (1974).

8. Van Der Hoek, L. et al. Identification of a new human coronavirus. Nat. Med.10, 368-373 (2004).

9. Woo, P. C. Y. et al. Characterization and Complete Genome Sequence of a Novel Coronavirus, Coronavirus HKU1, from Patients with Pneumonia. J. Virol.79, 884-895 (2005).

10. Ksiazek, T. G. et al. A Novel Coronavirus Associated with Severe Acute Respiratory Syndrome. N. Engl. J. Med.348, 1953-1966 (2003).

11. Drosten, C. et al. Clinical features and virological analysis of a case of Middle East respiratory syndrome coronavirus infection. Lancet Infect. Dis.13, 745-751 (2013).

12. Lu, R. et al. Genomic characterisation and epidemiology of 2019 novel coronavirus: implications for virus origins and receptor binding. Lancet395, 565-574 (2020).

13. Lim, Y., Ng, Y., Tam, J. \& Liu, D. Human Coronaviruses: A Review of Virus-Host Interactions. Diseases4, 26 (2016).

14. Shereen, M. A., Khan, S., Kazmi, A., Bashir, N. \& Siddique, R. COVID-19 infection: Origin, transmission, and characteristics of human coronaviruses. J. Adv. Res.24, 91-98 (2020).

15. Gretebeck, L. M. \& Subbarao, K. Animal models for SARS and MERS coronaviruses. Curr. Opin. Virol.13, 123-129 (2015). 
16. Tahamtan, A. \& Ardebili, A. Real-time RT-PCR in COVID-19 detection: issues affecting the results. Expert Rev. Mol. Diagn.20, 453-454 (2020).

17. Udugama, B. et al. Diagnosing COVID-19: The Disease and Tools for Detection. (2020) doi:10.1021/acsnano.0c02624.

18. Lisboa Bastos, M. et al. Diagnostic accuracy of serological tests for covid-19: systematic review and meta-analysis. BMJ370, 2516 (2020).

19. Alharbi, S. A., Almutairi, A. Z., Jan, A. A. \& Alkhalify, A. M. Enzyme-Linked Immunosorbent Assay for the Detection of Severe Acute Respiratory Syndrome Coronavirus 2 (SARS-CoV-2) IgM/lgA and $\lg G$ Antibodies Among Healthcare Workers. (2020) doi:10.7759/cureus.10285.

20. US Department of Health and Human Services, F. EUA Authorized Serology Test Performance About this page Test Performance Abbott Alinity i SARS-CoV-2 IgG. 1-17 (2020).

21. Liu, S. L. et al. Single-Virus Tracking: From Imaging Methodologies to Virological Applications. Chem. Rev.120, 1936-1979 (2020).

22. Conti, M. et al. Building up spectral libraries for mapping erythrocytes by hyperspectral dark field microscopy. Biomed. Spectrosc. Imaging5, 175-184 (2016).

23. Zamora-Perez, P., Tsoutsi, D., Xu, R. \& Rivera-Gil, P. Hyperspectral-enhanced dark field microscopy for single and collective nanoparticle characterization in biological environments. Materials (Basel).11, 1-19 (2018).

24. Peña, M. D. P. S. et al. Hyperspectral imaging of nanoparticles in biological samples: Simultaneous visualization and elemental identification. Microsc. Res. Tech.79, 349-358 (2016).

25. Fairbairn, N., Christofidou, A., Kanaras, A. G., Newman, T. A. \& Muskens, O. L. Hyperspectral darkfield microscopy of single hollow gold nanoparticles for biomedical applications. Phys. Chem. Chem. Phys.15, 4163-4168 (2013).

26. Alafeef, M., Moitra, P., Dighe, K. \& Pan, D. Hyperspectral Mapping for the Detection of SARS-CoV-2 Using Nanomolecular Probes with Yoctomole Sensitivity. ACS Nano (2021) doi:10.1021/ACSNANO.1C05226.

27. Loo, S. L. et al. Human coronaviruses 229E and OC43 replicate and induce distinct antiviral responses in differentiated primary human bronchial epithelial cells. Am. J. Physiol. - Lung Cell. Mol. Physiol.319, L926-L931 (2020).

28. Polson, A., Keen, A., Sinclair-Smith, C. \& Furminger, I. G. S. Polyethylene glycol purification of influenza virus with respect to aggregation and antigenicity. J. Hyg. (Lond).70, 255 (1972).

29. Gias, E., Nielsen, S. U., Morgan, L. A. F. \& Toms, G. L. Purification of human respiratory syncytial virus by ultracentrifugation in iodixanol density gradient. J. Virol. Methods147, 328 (2008).

30. KR, Y., BM, A., R, B., L, L. \& G, T. Rapid bacteriophage sedimentation in the presence of polyethylene glycol and its application to large-scale virus purification. Virology40, 734-744 (1970).

31. D. Lewis, G. \& G. Metcalf, T. Polyethylene Glycol Precipitation for Recovery of Pathogenic Viruses, Including Hepatitis A Virus and Human Rotavirus, from Oyster, Water, and Sediment Samples. Appl. 
Environ. Microbiol.54, 1983-1988 (1998).

32. Elden, L. J. R. van et al. Frequent Detection of Human Coronaviruses in Clinical Specimens from Patients with Respiratory Tract Infection by Use of a Novel Real-Time Reverse-Transcriptase Polymerase Chain Reaction. J. Infect. Dis.189, 652 (2004).

33. Roth, G. A., Tahiliani, S., Neu-Baker, N. M. \& Brenner, S. A. Hyperspectral microscopy as an analytical tool for nanomaterials. Wiley Interdisciplinary Reviews: Nanomedicine and Nanobiotechnology vol. 7 565-579 (2015).

34. More, S. S. \& Vince, R. Hyperspectral Imaging Signatures Detect Amyloidopathy in Alzheimer's Mouse Retina Well before Onset of Cognitive Decline. (2014) doi:10.1021/cn500242z.

35. Cancio, L. C. et al. Hyperspectral imaging: A new approach to the diagnosis of hemorrhagic shock. J. Trauma - Inj. Infect. Crit. Care60, 1087-1095 (2006).

36. Blanco, F. et al. Hyperspectral imaging based method for fast characterization of kidney stone types. J. Biomed. Opt.17, 076027 (2012).

37. Moe, O. W. Kidney stones: Pathophysiology and medical management. Lancet367, 333-344 (2006).

38. Nayir, A. Determination of urinary calculi by binocular stereoscopic microscopy. Pediatr. Nephrol.17, 425-432 (2002).

39. Lehmann, C. A., McClure, G. L. \& Smolens, I. Identification of renal calculi by computerized infrared spectroscopy. Clin. Chim. Acta173, 107-116 (1988).

40. D, W., A, M. \& MV, Y. Point-of-Use Nanobiosensor for Detection of Dengue Virus NS1 Antigen in Adult Aedes aegypti: A Potential Tool for Improved Dengue Surveillance. Anal. Chem.90, 679-684 (2018).

41. Padoan, R., Steemers, T., Klein, M. \& Aalderink, B. Quantitative Hyperspectral Imaging of Historical Documents: Technique and Application. ART Proc. 25-30 (2008).

42. Hsieh, W.-Y. et al. Single-Walled Carbon Nanotubes Induce Airway Hyperreactivity and Parenchymal Injury in Mice. doi:10.1165/rcmb.2011-00100C.

43. Chou, C.-C. et al. Single-Walled Carbon Nanotubes Can Induce Pulmonary Injury in Mouse Model. (2008) doi:10.1021/nl0723634.

44. Tabet, L. et al. Adverse effects of industrial multiwalled carbon nanotubes on human pulmonary cells. J. Toxicol. Environ. Health. A72, 60 (2009).

45. Luanpitpong, S., Wang, L. \& Rojanasakul, Y. The effects of carbon nanotubes on lung and dermal cellular behaviors. doi:10.2217/nnm.14.42.

46. Sanpui, P. et al. Single-walled carbon nanotubes increase pandemic influenza A H1N1 virus infectivity of lung epithelial cells. Part. Fibre Toxicol.11, 1-15 (2014).

47. Moitra, P. et al. Selective Naked-Eye Detection of SARS-CoV-2 Mediated by N Gene Targeted Antisense Oligonucleotide Capped Plasmonic Nanoparticles. ACS Nano14, 7617-7627 (2020).

48. LeCun, Y., Bengio, Y. \& Hinton, G. Deep learning. Nat. 2015 5217553521, 436-444 (2015).

49. Krizhevsky, A., Sutskever, I. \& Hinton, G. E. ImageNet Classification with Deep Convolutional Neural Networks. Commun. ACM60, (2017). 
50. Goodfellow, I. J. et al. Generative Adversarial Nets.

51. Wang, D. et al. early Detection of tomato spotted Wilt Virus by Hyperspectral Imaging and outlier Removal Auxiliary Classifier Generative Adversarial Nets (OR-AC-GAN). doi:10.1038/s41598-01940066-y.

52. Zamora-Perez, P. et al. Hyperspectral-enhanced dark field analysis of individual and collective photoresponsive gold-copper sulfide nanoparticles. Nanoscale13, 13256-13272 (2021).

53. Fakhrullin, R., Nigamatzyanova, L. \& Fakhrullina, G. Dark-field/hyperspectral microscopy for detecting nanoscale particles in environmental nanotoxicology research. Sci. Total Environ.772, 145478 (2021).

\section{Figures}
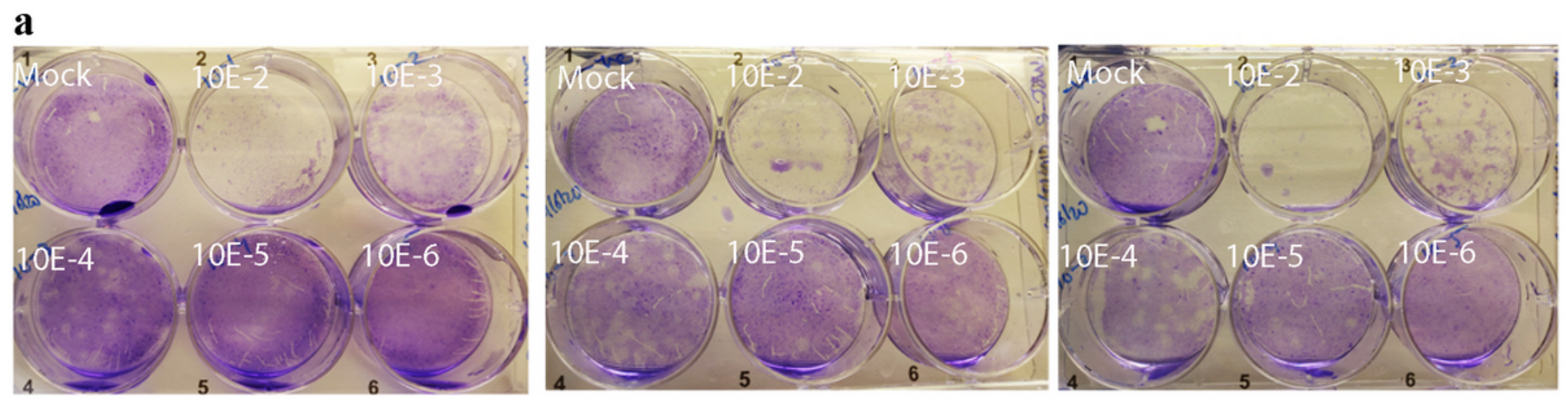

\section{b}
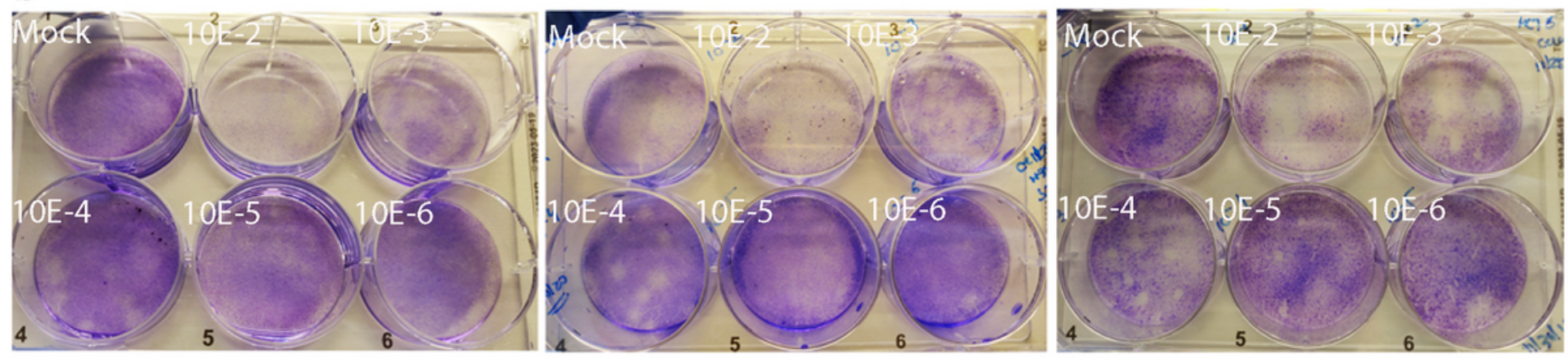

\section{Figure 1}

Plaque assay for assessment of $\mathrm{HCoV}$ viral titers. Plaques were observed at 11 days post-infection (dpi) with (a) HCoV-OC43 on a monolayer of HCT-8 and (b) 5 dpi with HCoV-229E on a monolayer of MRC-5. All the assays were performed in triplicates in a 6-well plate with a 10-fold serial dilution of viral stocks (indicated on top). Mock infected cells were included as controls. 

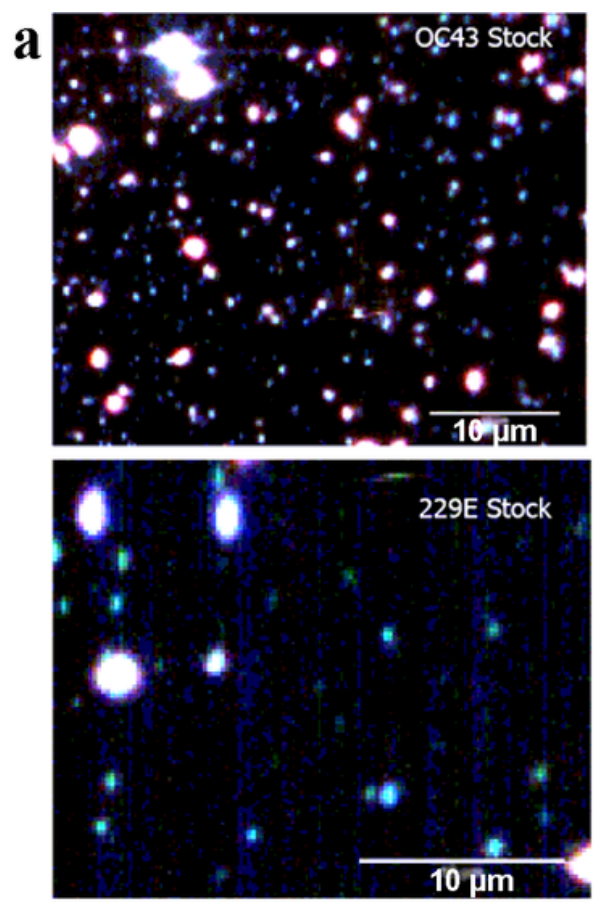
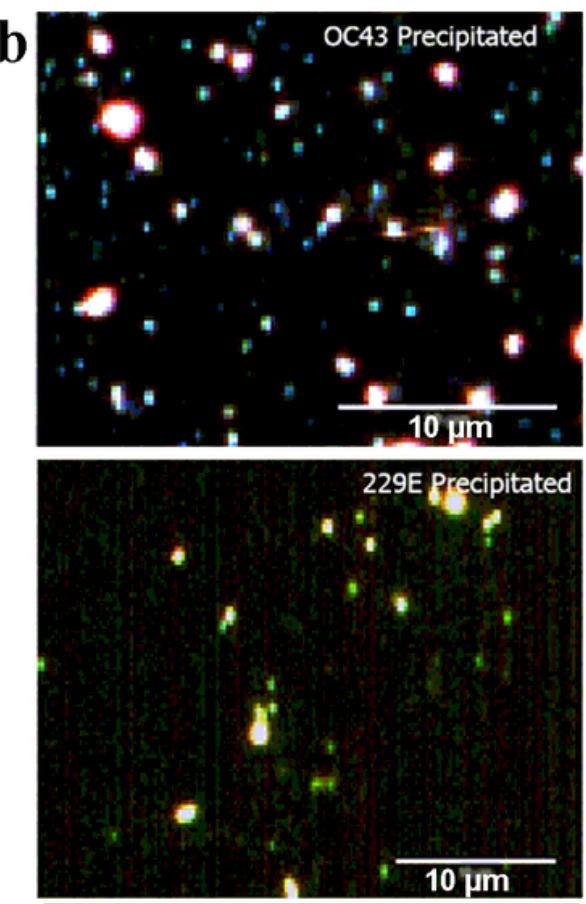

d

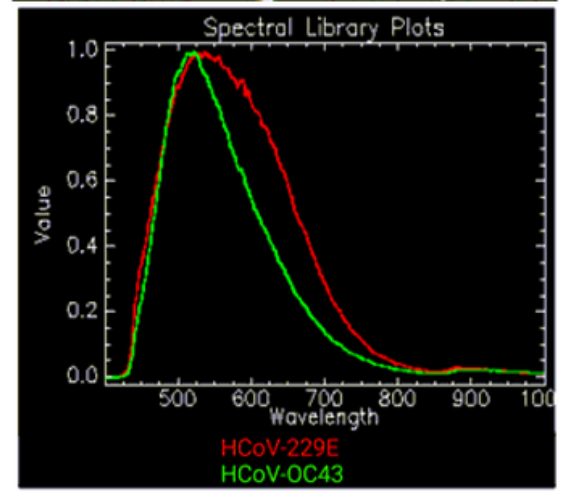

c
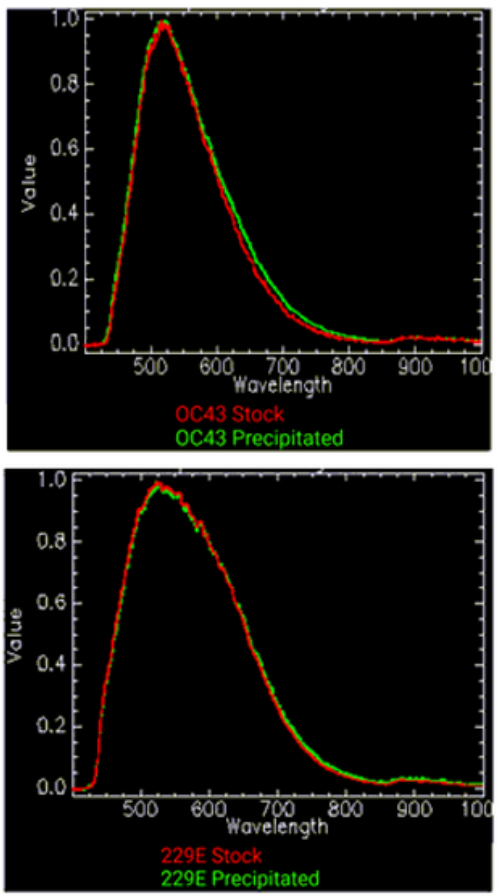

\section{Figure 2}

(a) EDHM imaging of commercially acquired viral stock of HCoV-OC43 (top) and HCoV-229E (bottom) viewed under 60X oil iris objective. (b) EDHM imaging of prepared viral stocks of HCoV-OC43 (top) and HCoV-229E (bottom). The white spots correspond to the viral particles. (c) The overlapping spectral profiles for HCoV-OC43 (top) and HCoV-229E (bottom) obtained commercially (red) and prepared by PEG precipitation (green). (d) Hyperspectral signal curves generated for HCoV-OC43 (green) and HCoV-229E (red) viral solution indicating a spectral peak difference of $\sim 30 \mathrm{~nm}$. 

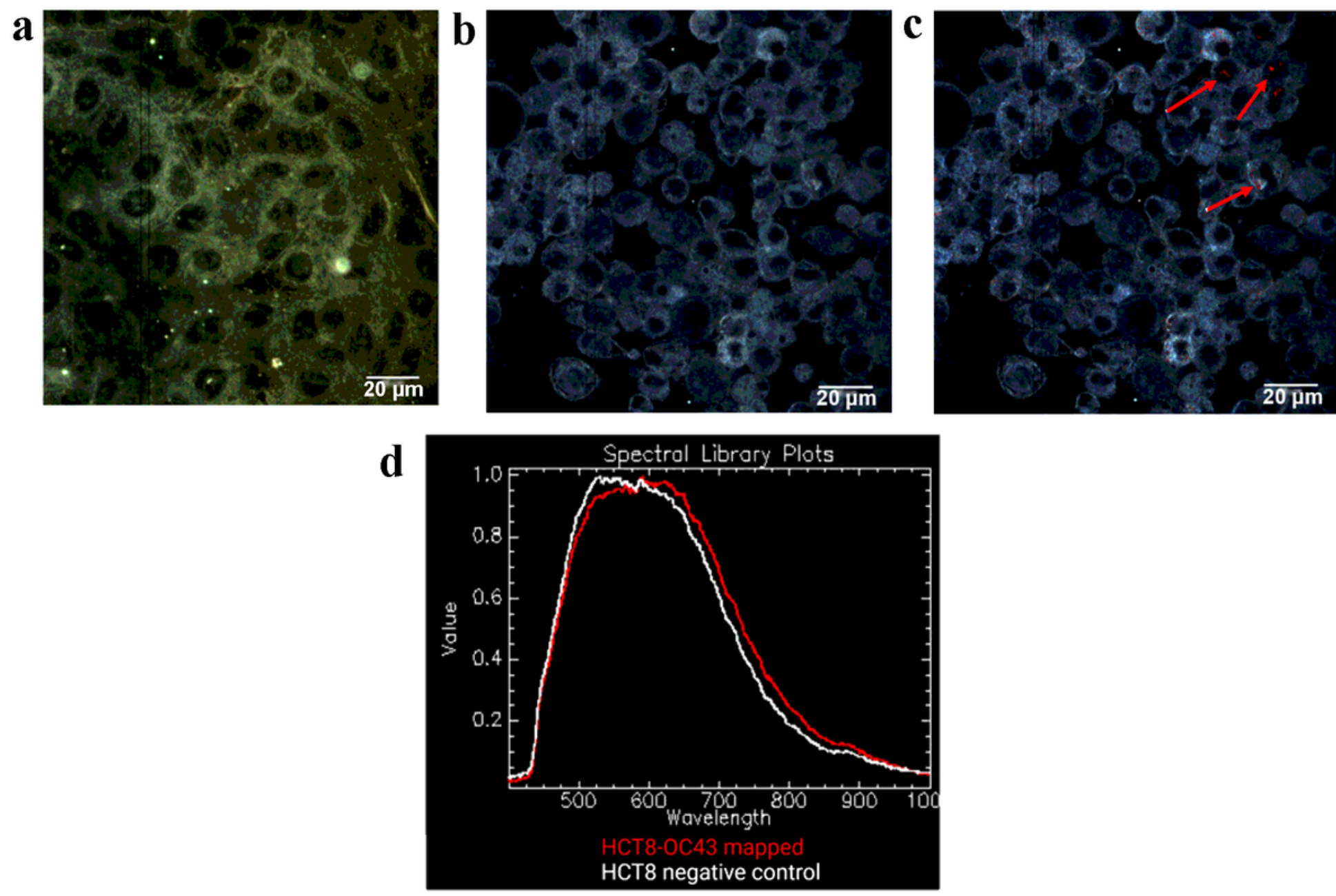

\section{Figure 3}

(a) EDHM imaging of uninfected HCT-8 cells and (b) HCT-8 cells infected with HCoV-OC43 visualized under $60 \mathrm{X}$ oil iris objective. (c) Spectral mapping resulted in the identification of pixels (red dots and indicating arrows) corresponding to viral particles in the infected HCT-8 cells. (d) Hyperspectral signal curves obtained for uninfected (white), and infected (red) HCT-8 cells show a peak shift of $\sim 50 \mathrm{~nm}$. 

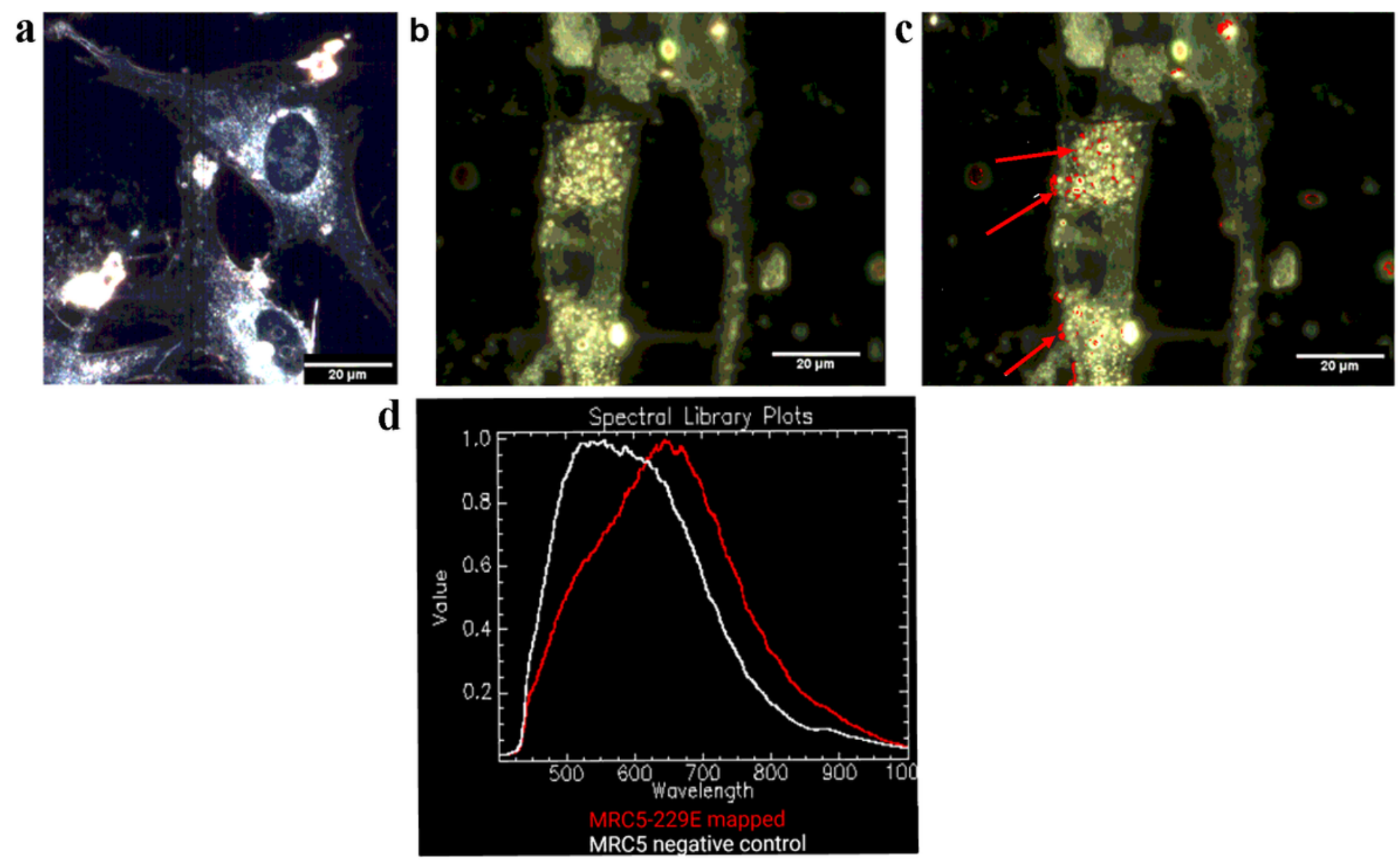

\section{Figure 4}

EDHM imaging of (a) uninfected and (b) infected MRC-5 cells with HCoV-229E visualized under $60 \mathrm{X}$ oil iris objective. (c) Spectral mapping of respective virus pixels in the infected MRC- 5 cells is indicated by red dots (red arrows). (d) Hyperspectral signal curves corresponding to uninfected (white), and infected (red) MRC- 5 cells show a peak shift of $\sim 150 \mathrm{~nm}$. 

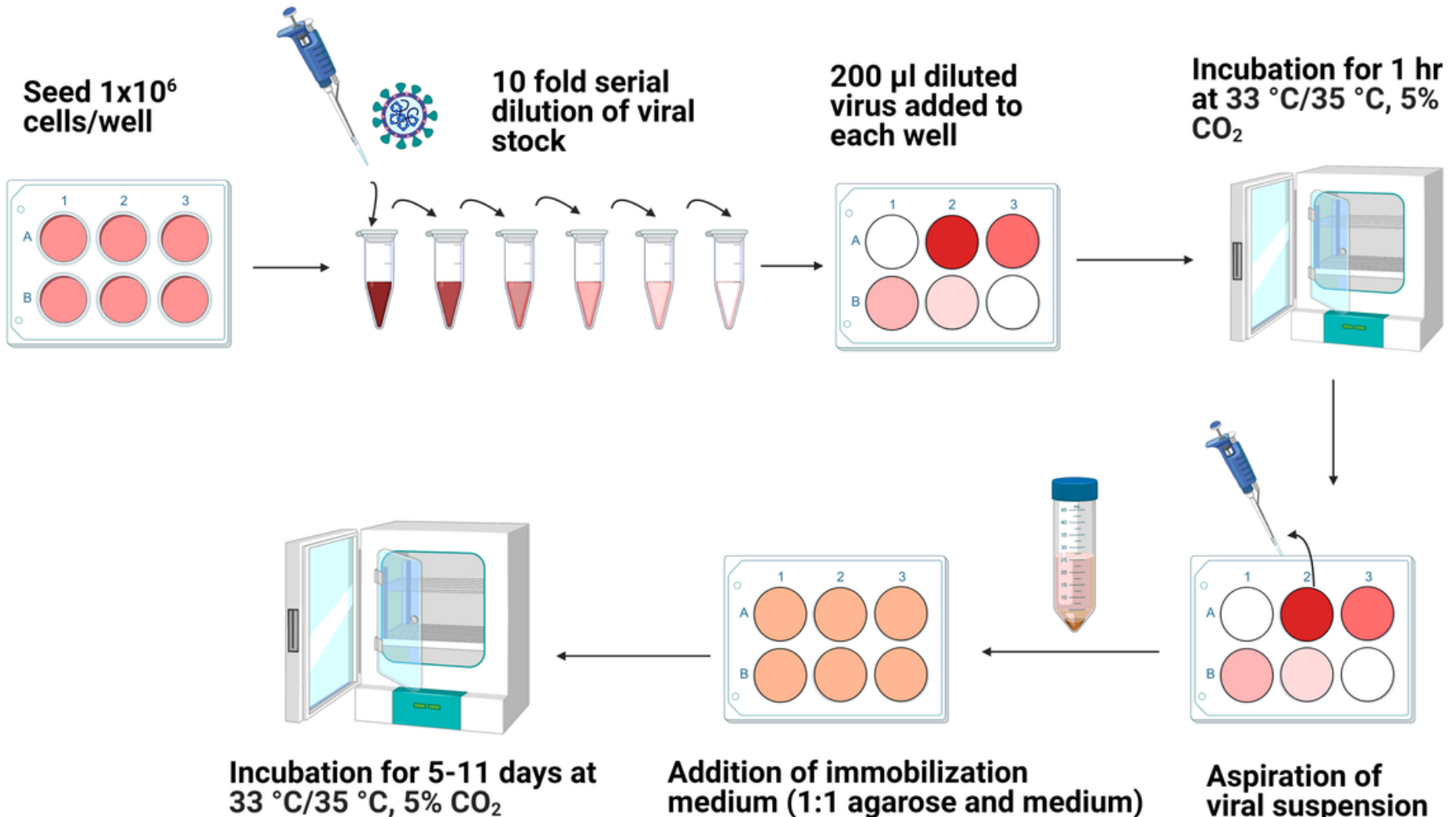

Incubation for 5-11 days at $33^{\circ} \mathrm{C} / 35^{\circ} \mathrm{C}, 5 \% \mathrm{CO}_{2}$ medium (1:1 agarose and medium)

\section{Aspiration of viral suspension}

Fixation and staining with
$1 \%$ crystal violet
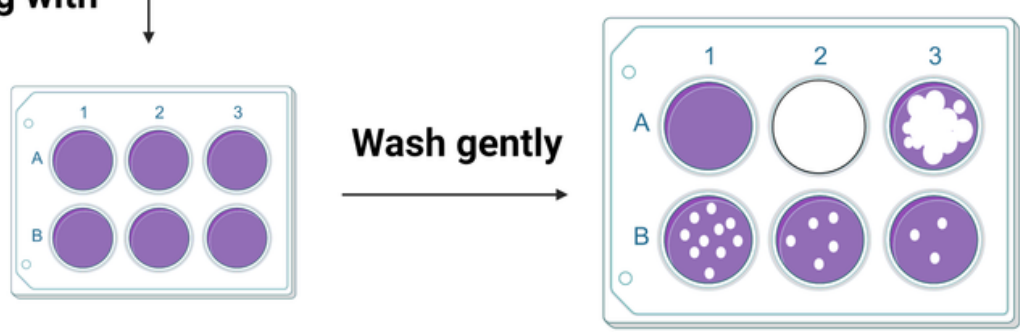

Plaque formation

Figure 5

Schematic representation of plaque assay procedure. The cells were grown to near full confluency in a 6well plate. The viral stocks were serially diluted and added to each well and incubated for $1 \mathrm{~h}$ at $33^{\circ} \mathrm{C}$ for $\mathrm{HCoV}-\mathrm{OC} 43$ and $35{ }^{\circ} \mathrm{C}$ for HCoV-229E in $5 \% \mathrm{CO}$. The viral suspensions were aspirated, and overlay media was added to each well. The plates were incubated for 11 days at $33^{\circ} \mathrm{C}$ in $5 \% \mathrm{CO} 2$ for $\mathrm{HCoV}-\mathrm{OC} 43$ infected cells or 5 days at $35{ }^{\circ} \mathrm{C}$ in $5 \% \mathrm{CO} 2$ for HCoV-229E infected cells. Post-infection, the cells were fixed and stained with $1 \%$ crystal violet followed by plaque counting. 